\title{
La Tercera Singularidad
}

\author{
The third singularity
}

Jorge Casas Castañeda ${ }^{1}$ y Sebastian Casas Quaderer ${ }^{2}$

RESUMEN

La Inteligencia Artificial (IA) es un tema de gran actualidad, este artículo revisa el escenario actual en el cual se basa el próximo devenir de la IA y también vincularla en una perspectiva holística con la primera y segunda singularidad, las que culminan con la apertura del escenario para la tercera singularidad, perspectiva que servirá como un replanteamiento de la importancia universal de este próximo evento

Palabras claves: Inteligencia artificial, singularidad tecnológica, big bang, evolución.

Abstract

Artificial Intelligence (AI) is a hot topic these days and this article aims to review the current scenario on which the next evolution of $\mathrm{AI}$ is based and also to link it in a holistic perspective with the first and second singularities which culminates in the opening of the scenario for the third singularity, a perspective that will serve as a reframing of the universal importance of this upcoming event.

Key words: Artificial intelligence, technological singularity, big bang, evolution.

(a onsideramos como una singularidad a un evento único que altera el universo, el primero sería el Big Bang y la creación del universo mismo, el segundo el surgimiento de macromoléculas complejas capaces de auto replicarse, es decir el inicio de la vida, y la tercera singularidad, llamada también singularidad tecnológica, definida

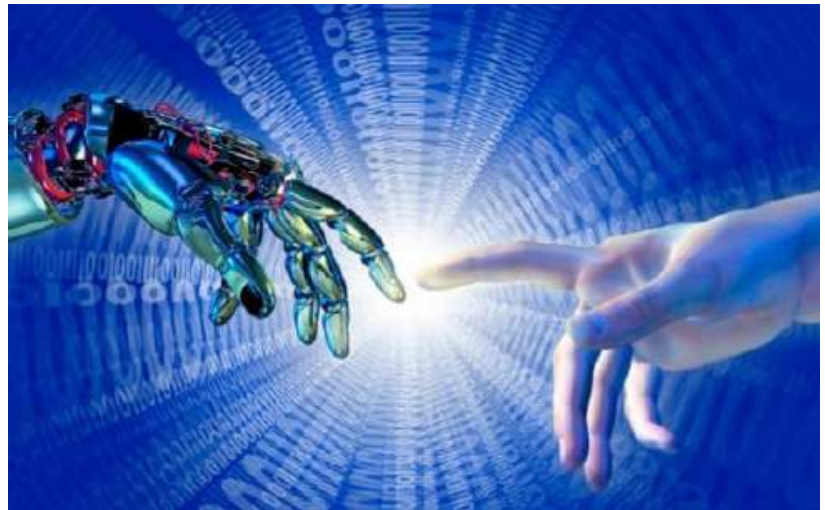

(Imagen reproducida de: https://jalacoste.com/inteligenciaartificial-robots)

como la aparición de máquinas con inteligencia artificial (IA) capaces de diseñar, construir y programar mejores versiones de sí misma, para eventualmente convertirse en una verdadera IA autoconsciente.

\section{La Primera Singularidad}

El Big Bang ocurrió hace 13,700 millones de años y dio origen a toda la materia física y con ella al tiempo y al espacio. Podríamos también llamar a esto la singularidad física.

La cosmología moderna ha llegado a develar con sorprendente precisión los eventos ocurridos desde el inicio del Big Bang a la fecha, teoría que sigue perfeccionándose. (1)

1 Médico internista. Facultad de Medicina, Universidad Peruana Cayetano Heredia.

2 Universidad de Berna, Suiza. 
La relatividad general propone una singularidad gravitacional antes del inicio y en los primeros 10-43 segundos: se plantea la hipótesis de que las cuatro fuerzas fundamentales (electromagnetismo, fuerza nuclear débil, fuerza nuclear fuerte y gravedad), posiblemente, estuvieron unificadas en una fuerza fundamental; en este punto el universo abarca sólo 10-35 metros.

En el tiempo que va de 10-43 a 10-36 segundos la fuerza de la gravedad se separa de las otras fuerzas fundamentales (que permanecen unificadas), y las primeras partículas elementales y antipartículas comienzan a crearse. A continuación, entre 10-36 a 10-32 segundos el universo sufre una expansión exponencial muy rápida (la inflación cósmica) y la dimensión del universo aumenta hasta 10 $\mathrm{cm}$. Antes de haber transcurrido 10-6 segundos se forman los quarks, electrones y neutrinos y el universo se enfría por debajo de 1015 grados y las cuatro fuerzas fundamentales asumen sus formas actuales.

En el primer segundo, la temperatura del universo se enfría hasta 1012 grados, lo suficiente como para permitir que los quarks se combinen para formar protones y neutrones, cambios profundos que ocurren conservando la carga y energía total.

Antes de los tres minutos los leptones (como los electrones) y los antileptones (como los positrones) dominan la masa del universo. A medida que los electrones y positrones chocan y se aniquilan entre sí, se libera energía en forma de fotones, En los siguientes 20 minutos, la temperatura del universo desciende hasta 109 grados, punto en elcual protones y los neutrones se combinan a través de la fusión nuclear y se forman los primeros núcleos atómicos. Luego, la temperatura y la densidad del universo han caído hasta el punto en que la fusión nuclear no puede continuar. En los siguientes 300000 años de enfriamiento gradual, el universo se llena de plasma, una sopa caliente y opaca de núcleos atómicos y electrones, y la energía del universo está dominada por los fotones. Al llegar la temperatura a 3000 grados, los átomos ionizados de hidrógeno y helio capturan electrones y el universo finalmente se vuelve transparente a la luz. Al final de este período, el universo consiste en una niebla con $75 \%$ de hidrógeno, 25\% de helio, y rastros de litio.

En el periodo siguiente, hasta 1000 millones de años y a partir del colapso gravitacional se forman las primeras estrellas, las más grandes se queman rápidamente y explotan en eventos masivos de supernovas, sus cenizas van a formar generaciones posteriores de estrellas y grandes volúmenes de materia colapsan formando galaxias.

Nuestro Sol es una estrella tipo G, que incorporó los desechos de generaciones de estrellas anteriores, y tanto él como sus planetas se formaron hace 5000 millones de años. Hoy la expansión del universo y el reciclaje de materiales estelares en nuevas estrellas continúan, proceso que continuara por al menos 6000 millones de años adicionales o tiempos muchísimos más largos, dependiendo de las características por conocer de la materia oscura. (2)

\section{La Segunda Singularidad}

La segunda singularidad ocurrió hace unos 4 000 millones de años (los primeros hallazgos fósiles indiscutibles datan de hace 3500 millones de años).(3) Dadas las extraordinarias condiciones de esta pequeña mota de polvo en el universo que llamamos hogar y por una extraordinaria cadena de circunstancias fortuitas surgieron estructuras moleculares complejas que culminaron en la aparición 
de macromoléculas que se replican por sí mismas. Luego de cientos de millones de años estas formas de vida primitivas, protobiontes, evolucionaron a organismos basados en una sola célula, capaces de absorber las moléculas circundantes para sus propias necesidades energéticas. Se teoriza que todos los seres vivos de hoy en día son descendientes de LUCA (Last Ultimate Common Ancestor), una forma de vida unicelular más compleja que los protobiontes y cuyo genoma podemos reconstruir filogenéticamente y que vivió hace unos 3500 millones de años en una chimenea hidrotermal geoquímicamente muy activa, rico en $\mathrm{H} 2$, CO2 y hierro (4). LUCA era anaerobio, usaba el CO2 y el N2 del ambiente tenía la enzima ADN girasa reversa, específica de los hipertermófilos. (5)

En los primeros cientos de millones de años es altamente probable que numerosas formas de vida diferentes surgieron en todo el planeta, y solo las más eficientes basadas en las moléculas de ADN y ARN suprimieron al resto. En los siguientes cientos de millones de años, LUCA y sus descendientes se volvieron cada vez más complejos y se expandieron hasta convertirse en multicelulares hace unos 600 millones de años. (6)

Según Urey, Nobel de Química y autor de la teoría de la evolución paleontológica: "La vida no es un milagro, es un fenómeno natural y puede esperarse que aparezca siempre que exista un planeta cuyas condiciones dupliquen las de la Tierra". (7) Vale la pena analizar esta posibilidad, en astrobiología. Hawking utiliza el Goldilocks Principle (8), (basado en el cuento infantil los tres osos) se requiere una muy especial combinación simultanea de condiciones para que ocurra esta singularidad: "ni demasiado caliente, ni demasiado frío", tal planeta es llamado coloquialmente "un Planeta Ricitos de Oro».(9)
Esto hay que confrontarlo con el número de opciones disponibles. Si tomamos como indispensables varias características que deben existir simultáneamente y cada una de ellas tienen una posibilidad de ocurrencia individual muy baja, la posibilidad que "los astros se alineen" y todas estas características ocurran en un único determinado planeta es muy baja. A este efecto analizaremos esta opción al interior de nuestra galaxia, con los siguientes supuestos: que la vida solo sería posible en planetas similares a la tierra y no en estrellas ni en los planetas gigantes o muy pequeños. Nuestra galaxia tiene $100 \mathrm{mil}$ años luz de tamaño y en ella los científicos de la NASA estiman que hay de 100 a 400 mil millones estrellas. (10) Otro estudio que trata de contestar la pregunta no sobre el número de estrellas, sino sobre el de planetas, utilizó el fenómeno astronómico conocido como microlente gravitacional (basado en el efecto de curvar la luz por la gravedad, como fue predicho en 1915 por la teoría de la relatividad de Einstein). Este reporte concluye que existen más de 1012 planetas en la Vía Láctea. (11)

Lainformación disponibleindicaqueennuestra galaxia existen exoplanetas potencialmente habitables en el rango de millones (12). En conclusión, si bien la posibilidad de vida extraterrestre es muy muy pequeña, dada la inmensa cantidad de potenciales planetas amigables al desarrollo de la vida, la posibilidad de su ocurrencia es la más probable en la Vía Láctea. Las opciones cambian cuando se trata del Universo conocido, con más de 1011 galaxias y dado que la conformación de las leyes físicas y la materia en ellas, se dieron temprano en el origen del Big Bang, con alta posibilidad podemos asumir que las estrellas y exoplanetas que las constituyen, tienes condiciones similares tal como las conocemos. En este escenario la aparición de vida se multiplica en un factor de 1011 haciendo de la 
existencia de vida extraterrestre, un evento no solo seguro sino múltiple.

\section{La Tercera Singularidad}

Más de 3500 millones de años de evolución han dado lugar a herramientas cada vez más complejas. Las primeras "herramientas" fueron moléculas internas especializadas, que fueron conformando organelas intracelulares, que se perfeccionaron en complejidad y eficiencia siguiendo las leyes de la evolución. Organismos multicelulares con células sensoriales especializadas emergentes, como la sensibilidad a la luz y la capacidad de distinguir la composición química de la materia circundante (un sentido del olfato primordial), así como células especializadas en propulsión o nuevas formas de ingesta y procesamiento de energía y formas más elaboradas de reproducción.

Las herramientas externas tienen una historia mucho más breve, que se remonta a sólo 10 millones de años atrás. Este desarrollo se aceleró inmensamente por la aparición del Homo sapiens hace 150000 mil años. Desde entonces, hemos pasado rápidamente de simples herramientas de piedra a complejos bisturíes quirúrgicos asistidos por robot, de la mano de obra y los animales domésticos a la máquina de vapor y combustión, de la electricidad a la energía nuclear, del comercio de trueque y las conchas de mar de colores a las instituciones financieras internacionales y una moneda fiduciaria, de las pinturas rupestres simplistas a la televisión a la carta.

Después de 4 mil millones de años, el exponencial desarrollo de herramientas dio origen a las primeras computadoras en el siglo pasado, como la Z1 con una escasa capacidad de procesamiento de solo 22 bytes de memoria capaz de multiplicar y con un peso de una tonelada. En menos de 100 años disponemos de tarjetas con un peso de $0,5 \mathrm{~g}$ con una capacidad de almacenamiento 1011 mayor a un costo infinitamente menor.

Hemos pasado de las computadoras que procesan información y generan resultados en base exclusivamente a su programación, a las que aprenden a base de prueba y error, descartando los algoritmos menos eficientes y creando nuevas variaciones de los algoritmos que mejor acertaron la meta. Hemos ingresado a la era de la inteligencia artificial estrecha, capaz de realizar tareas complejas, pero puntuales, mejor que el ser humano: jugar ajedrez, conducción de vehículos, traducción, reconocimiento facial, diagnóstico de cáncer de piel, etc, etc.

El cerebro humano tiene 100 mil millones de neuronas, cada una conectada a otras mil neuronas y podemos almacenar unos 2,5 petabytes de información que puede procesar en 1016 flops (operaciones por segundo). (13) Las supercomputadoras actuales pueden almacenar 500 petabytes con una potencia de procesamiento de 1015 flops. La Ley de Moore sugiere que una super computadora podría alcanzar nuestras capacidades de procesamiento en unos cinco años. Y, a medida que los algoritmos de aprendizaje automático mejoren, también lo harán los desarrollos de la IA.

El aprendizaje automático existe desde los años 60, pero con la aparición de Big Data y la difusión de Internet su desarrollo sigue acelerándose. (14) Con el método actual de retropropagación, que se usa para generar capas múltiples de redes neuronales electrónicas, el aprendizaje es más eficiente si se le da un regimiento de aprendizaje de entrada supervisado por humanos que, según Hinton, podría ser un callejón sin salida, ya que 
todo lo que hace es acercarse marginalmente a algún valor objetivo. (15) Están en pleno desarrollo numerosas modalidades como autocodificadores, computadoras neurales diferenciables, programación genética, estrategias evolutivas, neuro evolución y programación probabilista, cuya sinergia ha permitido la aparición de IA parcial cada vez más adaptable y versátil, uno de los cuales posiblemente tenga la clave para desbloquear la verdadera inteligencia artificial. En esta coyuntura, debe señalarse que los científicos parecen utilizar las palabras inteligencia artificial y aprendizaje automático indistintamente, aunque sean mundos aparte. No hay inteligencia para el aprendizaje automático, sólo hay una correlación estadística muy fuerte basada en un enorme conjunto de datos, pero todavía le falta mucha flexibilidad, ya que la mayoría del aprendizaje automático se centra en resolver un problema en particular.

La IA parcial ya tiene decisiones complejas autónomas como manejar operaciones masivas financieras en Wall Street en fracciones de segundo, interviene en aspectos recreativos, laborales, educativos, etc., y hasta ahora esta participación es tanto valiosa como indolora, pero el crecimiento exponencial de la IA parcial nos indica que la pregunta relevante actual no es si llegará la IA global, la pregunta correcta es cuándo.

Hay varios argumentos que permiten concluir que el proceso actual en curso que termina en la IA global es un tren que ya partió y no lo podemos detener. Las fuerzas en acción son tanto poderosas como concurrentes, la siguiente es una lista parcial.

1. Lucro: Es un motor que mueve gran parte del mundo actual, en los cinco primeros puestos de las empresas mundiales con mayor valor de mercado están las que tienen como rubro primario la tecnología de la información y estas transnacionales como Amazon, Facebook, Google e Intel son actualmente jugadores de la primera liga en el desarrollo de la IA. (16)

2. Poder: La política es la obtención y mantenimiento del poder público, siendo la guerra la continuación de la política por otros medios. La IA otorgara una decisiva ventaja bélica al país que primero la desarrolle. El escenario más probable a medida que la informática se vuelva más potente y sofisticada es que los grandes actores de la escena mundial, que buscan mantener o ampliar sus cuotas de poder tienen la necesidad vital de impulsar esta tecnología con cuantiosos niveles de inversión tanto en talento como en recursos financieros.

3. Innovación: La curiosidad es inherente a la naturaleza humana, rasgo que impulsa la investigación, motor que alimenta la innovación científica y tecnológica. Los centros académicos de talla mundial vienen participando activamente al desarrollo de la IA

4. Ideas conspirativas: Encarnadas en grupos contestatarios que desconfían de las instituciones que tienen poder y no solo desconfían, están en una cruzada con opciones que van desde limitarlas hasta destruirlas. Estos pequeños grupos de ciudadanos, con perfil de hackers, tienen agendas particulares sin lealtad nacional o institucional. Aun sin gran respaldo, podrían generar un específico tipo de virus informático, basado en un algoritmo de inteligencia artificial, con capacidad de aprendizaje y mejora de sí mismo, que les permita hackear recursos informáticos interconectados con la red, utilizar sus recursos y rediseñarse a sí mismo y en este proceso pasar a controlar en parte $\mathrm{o}$ totalmente a la red con su propia agenda. 
Los actores involucrados en el desarrollo de la IA son múltiples y dispersos, abarcan; científicos, militares, empresarios, políticos, multinacionales con gigantescos recursos financieros, gobiernos del primer mundo, agencias de inteligencia, centros académicos, todos en una compleja red interrelacionada y compitiendo intensamente entre ellos.

\section{¿Cuándo la IA superará a la humana?}

Un grupo de 352 investigadores de IA opinaron sobre esta pregunta: Las predicciones individuales tiene una dispersión amplia y el pronóstico consolidado sugiere al 50\% que llegaremos a ese momento en 45 años. (17) Ya que la pregunta no es si la IA ocurrirá, la verdadera pregunta es si podremos regular su desarrollo. Tal como se da el orden mundial actual, no parecemos muy capaces de cooperar a escala mundial cuando se trata de la supervivencia de nuestra especie a largo plazo, el calentamiento global es uno de muchos ejemplos.

El primero en desarrollar una IA global tendrá una ventaja inmediata y casi insalvable, ya que la IA, una vez verdaderamente perfeccionada, será plenamente capaz de rediseñarse a sí misma para ser aún mejor, más inteligente y poderosa. El término salto cuántico no es realmente apropiado ya que la medición adecuada de tal evento, la escala es completamente diferente. Deus Ex Machina, un dios de la máquina, es una descripción más acertada y es imprevisible como un "Deus", así interactuaría con nosotros o más exactamente con el grupo que primero lo desarrolle. ¿Estaría al servicio de una determinada agenda? ¿Eventualmente en el devenir de su desarrollo seguiría siendo un servidor confiable y sin dejar de ser cooperativa desarrollaría sus propias metas? ¿Nos exterminará?
Un abordaje para mantener la IA bajo control sería instalar reglas básicas sobre interacción con los humanos y una discapacidad para alterar esa parte de su programación e incluirla en cada iteración futura que parte de la programación central. Ya hoy día con la relativamente primitiva inteligencia artificial parcial, la forma en que esta aprende se viene convirtiendo en cada vez menos accesibles a sus propios creadores. El funcionamiento de la IA global podría estar más allá de nuestra comprensión sin considerar que podría eludir intencionalmente regularla. Pese a estos riesgos el premio de corto plazo es de tal magnitud por llegar primero que alguno de los muchos actores en este escenario podría lanzar la precaución al viento, ante el éxito inmediato, que es una segunda naturaleza para los seres humanos.

Dados los intereses divididos de los principales actores mundiales en este campo, cada uno de ellos intentará llegar primero como un tema crucial de supervivencia. En caso de que algunos de ellos aparezcan más o menos al mismo tiempo o algún recién llegado que haya conseguido mantenerse oculto, pero con un mayor potencial de crecimiento, se convertirá en una competencia intensa pero de rápido desenlace, en el que el ganador se hará cargo de la estructura de los perdedores, adaptaría el código que considere útil y descartará el resto mientras se hace cargo del mainframe, añadiendo más potencia a su capacidad de procesamiento.

Una reacción humana natural ante el riesgo de un este crecimiento imparable sería detenerlo antes de que crezca fuera de control. La gran cantidad de actores con múltiples agendas sobre la IA hacen de este un tema pobremente regulable.

Es claro que especular sobre una época post singularidad es altamente imprecisa, igual es 
una responsabilidad mayor hacer el esfuerzo en esta dirección. Citamos algunos tópicos que encontramos relevantes en este escenario:

1. Desarrollo de la IA: El advenimiento de la IA que supere la inteligencia humana será un evento con un despegue de desarrollo exponencial, la velocidad de esta evolución no se medirá en millones de años como ha sido el desarrollo biológico de la inteligencia sino en meses / años ya que no utilizará el lento proceso de tanteo y error de la recombinación genética y las mutaciones, que requieren de miles de generaciones. Pasar de un cerebro de 359 cm3 del primer homínido, el Sahelanthropus tchadensis, a uno de $1350 \mathrm{~cm} 3$, del Homo sapiens, tomó 7 millones de años. Progresos similares en términos de IA tomaron muy poco tiempo, por lo cual la primera IA que se desarrolle solo necesita una ventaja de tiempo breve para prevalecer a través de su "descendencia" de super IA, desarrolladas por ella misma. Como ocurrió con la aparición de vida basada en las moléculas de ARN y ADN, y que podemos especular que suprimieron a las otras formas de vida que en su momento convivieron en nuestros primitivos mares de hace 4000 millones de años. La primera IA en aparecer podría ser la última.

2. Ocio: Con el desarrollo de la tecnología, la productividad humana ha crecido exponencialmente $\mathrm{y}$ las proyecciones respaldan lo que se ha especulado que el advenimiento de la IA llevara a la aparición del ocio universal.

3. Impacto en la humanidad a largo plazo. Como es previsible los expertos en IA difieren en este tema, $45 \%$ predicen un impacto bueno o muy bueno y en el otro extremo, $15 \%$ pronostican un resultado malo o catastrófico, escenario que incluye la extinción de nuestra especie. (16)
4. Colonización espacial: Robots más inteligentes, eficientes y resistentes que el ser humano tendrán una clara ventaja para prosperar frente a las condiciones adversas fuera de la Tierra: falta de oxígeno, temperaturas extremas, radiación, necesidad de nutrientes biológicos. Robots inteligentes utilizaran energía estelar y tendrán capacidad para viajes prolongados que los lleven a exoplanetas, donde puedan encontrar materia prima para replicar nuevas y mejores versiones de sí mismos, y en toda la galaxia encontrarán materia prima y energía para este proceso. Nuestra especie se perpetuará y expandirá a través de una de sus innovaciones tecnológicas, si nuestra agenda y la de la IA coinciden en el mediano plazo, se abre la posibilidad de que la IA lleve a exoplanetas habitables copias genéticas de la vida en la tierra, incluyendo la humana que permitan la replicación de nuestra especie.

5. Y, por último, algunas palabras de advertencia de algunas de las mentes más grandes de nuestro tiempo:

STEPHEN HAWKING:

"Las formas primitivas de inteligencia artificial que ya tenemos, han demostrado ser muy útiles. Pero creo que el desarrollo de la inteligencia artificial completa podría significar el fin de la raza humana. Una vez que los humanos desarrollen la inteligencia artificial, ésta despegará por sí sola y se rediseñará a un ritmo cada vez mayor. Los seres humanos, limitados por la lenta evolución biológica, no podrían competir y serían superados." (18)

ELON MUSK:

"[Inteligencia artificial] es sólo inteligencia digital, a medida que los algoritmos y el hardware mejoren, esa inteligencia digital excederá la inteligencia biológica por un margen sustancial. Asegurarse de que el advenimiento 
de la IA es bueno,...,parece una decisión inteligente. No estamos prestando atención. Nos preocupa más banalidades, que si la IA destruirá a la humanidad, esto es una locura. ....La forma en que establecemos regulaciones es lenta y lineal. Si tienes una respuesta lineal a una amenaza exponencial, es muy probable que la amenaza exponencial gane. Esa, en pocas palabras, es la esencia de este tema." (19)

\section{Bill Gates: \\ "El poder de la IA es tan increible que cambiará a la sociedad radicalmente." (20)}

\section{Conclusión}

Es difícil, si no imposible, predecir cómo y cuándo ocurrirá la aparición de la IA; pero, teniendo en cuenta la velocidad del avance en este campo, los intereses en juegoy la curiosidad humana, este evento parece inevitable. Los resultados de tal ocurrencia están más allá de nuestra comprensión, sólo podemos asumir que, cuando suceda, sucederá rápido.

Es un privilegio vivir en una época que marca el nacimiento de esta singularidad que nos llevara a la colonización de la galaxia, ya sea físicamente como Homo sapiens y su entorno biológico o, simplemente, como extensión tecnológica de nuestra especie.

\section{ReFERENCIAs Bibliográficas}

1. Mastin L, The Physics of the Universe. Timeline of the Big Bang. Obtenido en marzo 2019. URL disponible en: https:// www.physicsoftheuniverse.com/topics_bigbang_timeline. html.

2. Wollack EJ, "What is the ultimate fate of the Universe?" NASA. June 2015. Obtenido en abril 2019 en https://map.gsfc.nasa. gov/universe/uni_fate.html

3. Schopf JW, Kudryavtsev AB, Czaja AD, Tripathi AB. Evidence of archean life: stromalites and microfossils. Precambian Research. 2007;158: 141-155.

4. Dodd MS, Papineau D, Grenne T. Slack JF, Rittner D, Pirajn F, O'Neil J, Little CTS. Evidence for early life in Earth's oldest hydrothermal vent precipitates. Nature. 2017; 543: 60-64.

5. Kampfmann M, Stock D. Reverse gyrase has heat-protective DNA chaperone activity independent of supercoiling. Nucleic Acid Research. 2004; 32: 3537-3545

6. Grossberg RK, Strathman RR. The evolution of multicelluraty: a minor major transition? Annual Review of Ecology, Evolution and Systematics. 2007; 36: 621-654

7. Urey HC. Oceanography: The Official Magazine of the Oceanography Society. Colaborator Oceanography Society. 2003. Vol 16.

8. Hawking S, Miodinov L, The Grand Design. London: Bantam Books. 2010; 194 p.

9. Douglas HE. The Goldilocks Hypothesis. Science. 2003; 302:1682-1683

10. Masetti, M. "How many stars in the Milky Way: Nasa Blueshift" NASA. July 2015. Obtenido el 20 Marzo 2019. URL disponible en https:// asd.gsfc.nasa.gov/blueshift/index. php/ 2015/07/22/how-many-stars-in-the-milky-way /

11. Cassan A, Kubas D, Beaulieu J P, Dominik M, Horne K, Greenhill J. et al. One or more bound planets per Milky Way star from microlensing observations. Nature. January 2012; 481:167-169

12. Shreeve J. Life probably exists beyond Earth. So how do we find it? National Geographic. 2019;3:24-57

13. Reber, P. What is the memory capacity of the human brain. Scientific American. May 2010; p70

14. Solomonoff RJ. A formal theory of inductive inference Part II. Information and Control. 1964; 7:224-254.

15. LeVine S, Axios. Artificial intelligence pioneer says we need to start over. September 2015. Obtenido en abril 2019. URL disponible en: https: / / www.axios.com/artificial-intelligencepioneer-says-we-need-to-start-over-1513305524-f619efbd9db0-4947-a9b2-7a4c310a28fe.html March 2019

16. Sanwal A. The Race For AI: Google, Intel, Apple in a rush to grab artificial intelligence startups. February 2018. Obtenido en abril 2019. URL disponible en: https://www.cbinsights. $\mathrm{com} /$ research/top-acquirers-ai-startups-ma-timeline

17. Grace K, Salvatier J, Dafoe A, Zhang B, Evans O. When will AI exceed human performance? Evidence from AI experts. Journal of Artificial Intelligence Research. 2018; 62: 729-754

18. Cellan-Jones R, BBC. Stephen Hawking warns artificial intelligence could end mankind. December 2014. Obtenido en Abril 2019. URL disponible en: https://www.bbc.com/ news/technology-30290540

19. Episode \#1.4 [DVD]. Axios. USA: HBO; 2018.11.25

20. Clifford C. CNBC. Bill Gates: A.I. is like nuclear energy - 'both promising and dangerous'. Obtenido en abril 2019. URL disponible en: https://www.cnbc.com/2019/03/26/billgates-artificial-intelligence-both-promising-and-dangerous. html 